\section{Research Square}

Preprints are preliminary reports that have not undergone peer review.

They should not be considered conclusive, used to inform clinical practice, or referenced by the media as validated information.

\title{
Validity of Procalcitonin as a Single Biomarker for Predicting Mortality in Pediatric Patients after Acute Traumatic or Post-Surgical Infections: A Retrospective Study
}

\author{
Waleed Albuali ( $\nabla$ wbuali@iau.edu.sa ) \\ Imam Abdulrahman Bin Faisal Univeristy/King Fahd Hospital of the University
}

\section{Research Article}

Keywords: Procalcitonin, mortality, pediatric intensive care unit, post-surgical infection

Posted Date: April 21st, 2021

DOI: https://doi.org/10.21203/rs.3.rs-424596/v1

License: (c) (i) This work is licensed under a Creative Commons Attribution 4.0 International License. Read Full License 


\section{Abstract}

\section{Background}

Procalcitonin (PCT) levels are elevated in pediatric patients with systemic inflammation. This study was aimed to evaluate the use of Procalcitonin as a single biomarker in the prediction of mortality in pediatric patients after acute traumatic events or post-surgical infection.

\section{Methods}

This retrospective longitudinal study involved pediatric patients aged more than two weeks who were admitted to Pediatric Intensive Care Unit (PICU) of King Fahd Hospital of the University from January 2017 to December 2019 due to post-surgical infection. The data includes age, gender, comorbidity, severity of trauma, Pediatric Risk of Mortality Score (PRISM III) score in 24h of admission, PCT level at 24h and 48-72h, day-5 admission, blood culture, PICU stay, multiorgan failure, number of inotropes and intubation requirement were retrieved.

\section{Results}

The mortalities after surgical infection was of $15.9 \%$. Significantly, the elevated mean levels including PRISM III score, PCT level at $24 \mathrm{~h}, 48-72 \mathrm{~h}$ and day-5 admission were found in patients who were expired during their hospital stay. The PCT level at $48-72 \mathrm{~h}$ was 0.89 (best cutoff point $>1.35$ ) revealed as highly predictive validity of PCT compared to PCT level at $24 \mathrm{~h}$ which was 0.76 (best cutoff point $>0.15$ ) respectively.

\section{Conclusion}

The serum PCT level as a single laboratory biomarker is remarkably useful in the prediction of mortality after surgical infection for commencing of an earliest treatment plan as a lifesaving attempt. A blended approach based on the PCT level with other clinical biomarkers can be developed for monitoring a treatment given.

\section{Background}

Incidence of post-surgical systematic inflammatory response syndrome (SIRS) in pediatric age group is persistently increase in spite of a continuous advancement in the sterilization of surgical equipment and environmental hygiene to perform invasive diagnostic and therapeutic procedures in high-resource countries like Saudi Arabia [1, 2]. In Intensive Care Unit (ICU), availability of an instant, accurate, and specific laboratory tests regardless of cost or invasiveness are extremely desired by clinicians in identifying infected patients when some specific gold standard is controversial, also deprives benefit from prompt empirical antibiotic therapy [3-5].

Use of biomarkers and predictive scoring is a novel approach to assess infection, make diagnosis, estimate disease severity and forecast of patient's outcome. At present, C-reactive protein (CRP) and procalcitonin (PCT) levels are widely used biomarkers in the routine clinical practice. Predictive mortality using PCT level at a cut-off value of $6.38 \mathrm{ng} / \mathrm{dl}$ has a sensitivity and specificity $81.8 \%$ and $80.8 \%$ respectively, ROC area $=0.838$, was the highest as compared to CRP and leucocyte [6], but predictive efficiency of PCT in detecting acute bacterial infection and severity of outcome in terms of sensitivity and specificity on specific cutoff values are still to be standardized. Another study consisting of 105 neurotrauma patients [7] reported prediction of mortality based on procalcitonin using area under ROC on admission was $0.76(p<0.05)$ and found it comparatively better than CRP area of ROC $=0.73(p<0.05)$.

Overall rate of sepsis in ICU admitted patients in hospital-based study in Saudi Arabia has described 16\% and of SIRS $8.1 \%$, whereas $40.3 \%$ mortality rate associated with sepsis was reported [8]. However, there is no single study reported about the predictive efficacy of PCT level in post-surgical sepsis and associated mortality in pediatric patients, except the recent study in the same set up in Saudi Arabia relevant to some extent where five years' experience of predictive mortality using procalcitonin level biomarker based on 400 patients admitted in PICU on mechanical ventilator was reported [9].

The present study was a step ahead to explore the use of procalcitonin as a biomarker in the prediction of mortality in pediatric patients after acute traumatic or post-surgical infection. An increased predictive value of PCT in the estimation of in-hospital outcome based on post-surgical events, data may provide a sufficient evidence to adopt procalcitonin biomarker as baseline standard procedure.

\section{Methods}

This was a retrospective longitudinal study based on a cohort of critically ill pediatric patients from our previous study participants [9] admitted in PICU of King Fahd Hospital of the University (KFHU) from January 2017 to December 2019 to evaluate predictive validity of biomarkers in prediction of in-hospital outcome in terms of survival or mortality. It includes pediatric patients older than two weeks to 14 years of age admitted in PICU due to significant trauma or post-acute surgical events having at least two of the following four criteria, one of which must be either abnormal temperature or leukocytes count: 1 ) Temperature of $>38.5^{\circ} \mathrm{C}$ or $<36^{\circ} \mathrm{C}, 2$ ) Tachycardia, defined as a mean heart rate $>2$ Standard Deviation (SD) above the normal for age, or bradycardia, defined as a mean heart rate $<10$ th percentile for age, 3 ) Mean respiratory rate $>2$ SD above the normal for age, or mechanical ventilation for an acute insult that is not related to an underlying neuromuscular disease or the administration of general anesthesia, 4) Elevated or depressed Leukocytes count for age (excluding chemotherapy-induced leukopenia) or $>10 \%$ immature neutrophils.

Patients admitted for $<24 \mathrm{~h}$, who died within the first $24 \mathrm{~h}$, those who experienced cardiac arrest before PICU admission, those admitted to the PICU due to burn or chemical trauma, and those with incomplete or missing data were excluded from this study. All clinical and laboratory data were registered. The type and severity of trauma was classified according to the Injury Severity Score (ISS). Predictive validity of biomarkers in the estimation of in-hospital outcome were compared. These data including age, gender, comorbidity, severity of trauma based on the ISS and Pediatric Risk of Mortality Score (PRISM) III score at 
$24 \mathrm{~h}$ of admission and PCT level at $24 \mathrm{~h} 48 / 72 \mathrm{~h}$ and 5 days of admission, blood culture, PICU stay, multi-organ failure, number of inotropes and intubation requirement were retrieved as per required outcomes.

Data analysis was performed through SPSS-20.0 (IBM product, Chicago-USA). All categorical data were presented as frequencies and percentages. Chi-square test or Fisher's exact test was used for comparison of proportions in relation to expired versus survived outcomes. Numeric data based on age, weight, PICU stay, mechanical ventilation stay and levels of biomarkers were presented as Mean \pm SD. Unpaired t-test in case of data distributed normally or non-parametric Wilcoxon Mann-Whitney U-test for non-Gaussian distribution were applied for comparison of numeric variables between expired versus survived outcomes. ROC analysis was performed to estimate predictive validity of PRISM-III and PCT level at $24 \mathrm{hr} 48 / 72$ hour and 5 days of admission. Kaplan Meier's survival analysis was performed to show probability of survival in relation to length of PICU stay and mechanical ventilator. P-value of $\leq 0.05$ was considered statistically significant.

\section{Results}

Total of 245 pediatric patients fulfilling inclusion criteria were reviewed, having the mean age of $5.27 \pm 4.20$ years. Male preponderance in the study (58.8\%). Sepsis was seen in 76 (31.0\%) patients. Total 39 (15.9\%) mortalities after surgical infection took place, out of them 22/39 (56.4\%) were diagnosed with sepsis. In-hospital outcome of the pediatric patient was non-significant with regards to gender $(p=0.461)$, need of mechanical ventilator $(p=0.371)$, but inhospital outcome was highly significant associated with type of etiology, blood culture positivity and comorbidity $(p<0.001)$ as presented in Table 1 .

Significantly elevated mean levels of biomarkers including PRISM III score, PCT at 24 h, PCT $48-72 \mathrm{~h}$ and PCT at day-5 were found in patients who were expired during the hospital stay $(\mathrm{p}<0.001)$. There were also significantly increased mean length of PICU stay, ventilation stay and number of inotropic drugs in expired than survived group $(p<0.001)$, except mean age and weight were significantly lesser in expired group $(p<0.05)$ as detailed in Table 2 .

Area under the curve (AUC) for PRISM III score was 0.99 (95\% Cl: 0.98-1.00), p< 0.001 revealed as highly significant predictive validity of PRISM III in estimation of predictive mortality on best cutoff point $>9.5$ where sensitivity, specificity, Positive Predictive Values (PPV) and Negative Predictive Values (NPV) of PRISM III score were $94.9 \%, 93.9 \%, 94.6 \%$ and $99 \%$ respectively. Whereas, area under ROC of PCT level at $24 \mathrm{~h}$ was 0.76 (95\% Cl: $0.66-0.85$ ), p<0.001 revealed highly significant validity of PCT in prediction of in-hospital mortality on the best cutoff point $>0.15$ where sensitivity, specificity, PPV and NPV of PCT level were $66.7 \%, 74.3 \%, 32.9 \%$ and $92.2 \%$ respectively shown in Fig. 1 (Comparison of predictive validity of PRISM and PCT on 24 hours, $48-72$ hours and PCT at day-5 to estimate patient's in-hospital poor outcome) and Table 3.

However, area under ROC of PCT level $48 / 72 \mathrm{~h}$ was 0.89 (95\% Cl: $0.85-0.93), \mathrm{p}<0.001$ that revealed highly significant predictive validity of PCT in estimation of in-hospital mortality on the best cutoff point $>1.35$ where sensitivity, specificity, PPV and NPV were $82.1 \%, 82.0 \%, 46.3 \%$ and $96 \%$ respectively. Area under ROC of PCT level at 5 days was 0.87 (95\% Cl: $0.81-0.92), p<0.001$ that revealed highly significant predictive validity of PCT in estimation of in-hospital mortality on the best cutoff point $>0.15$ where sensitivity, specificity, PPV and NPV were $74.4 \%, 71.4 \%, 33 \%$ and $93.7 \%$ respectively shown in Fig. 2 (Comparison of predictive validity of PCT level on 48/72 hours and day-5 to estimate patient's in-hospital poor outcome) and Table 4.

The mean survival time in PICU was 24.6 (95\% Cl: 22.8-26.3) days, highest probability of mortalities (90\% or above) within first 15 days shown in Fig. 3 (Survival analysis of pediatric patients in pediatric ICU). The mean survival time on mechanical ventilator was 16.8 (95\% Cl: 15.4-18.2) days, highest probability of mortalities (95\% or above) within first 7-8 days shown in Fig. 4 (Survival analysis of pediatric patients in mechanical ventilation).

\section{Discussion}

Total 39 (15.9\%) mortalities after surgical infection took place, out of them 22/39 (56.4\%) were diagnosed with sepsis, which is comparatively higher than that reported in local study [8], also higher than mortality rates reported in a systematic review [10], 44\% mortality rate was the highest [11]. Turkish study reported highest predictive validity of procalcitonin (PCT) than C-reactive protein (CRP) and leukocyte in prediction of mortality in critically ill patients in PICU, reported area under ROC was $0.838(0.711-0.966)$, sensitivity and specificity at best cutoff point $6.38 \mathrm{ng} / \mathrm{ml}$ were $81.8 \%$ and $80.8 \%$ respectively [6], which was consistent with our findings of PCT at $48 / 72 \mathrm{~h}$ the sensitivity and specificity were respectively $82.1 \%$ and $82.0 \%$, even AUC $=0.89$ (95\% Cl: $0.85-0.93)$ in our study was higher.

However, sensitivity and specificity of PCT level at $24 \mathrm{~h}$ were $66.7 \%, 74.3 \%$ in this study were comparatively low as well as the area under ROC $=0.76(95 \% \mathrm{Cl}$ : $0.66-0.85$ ), the best cutoff point $>0.15$. Likewise results were reported in a study based on neuro-traumatic adult patients done by Deng et al [7], areas under the ROC at admission for the prediction of mortality was 0.76 . The findings were also remarkably higher than that reported by Masson et al [12], who compared PCT with Presepsin for mortality prediction in sepsis and exhibited presepsin as better prognostic biomarker than PCT in the range of Sequential Organ Failure Assessment score (area under the curve (AUC) from 0.64 to 0.75 vs. AUC 0.53 to 0.65 ). Another study [13] on adult patients in ICU following multiple trauma, initial PCT yielded AUC $=0.805$.

This study exhibited significantly elevated mean levels of biomarkers including PRISM III level, PCT at 24 h, PCT $48-72$ h and PCT at day-5 of those patients with poor outcome $(p<0.001)$, which is consistent with the findings of all studies included in the review article [14-16]. After pediatric liver transplant (PLT) observed serial PCT measurements during the first week was not useful to identify patients with bacterial infections, but they proposed that serum PCT may be useful after the first week post PLT [17]. The evidence support that procalcitonin can provide invaluable information when viewed as one piece of a clinical puzzle, and is most powerful when the interpreting physician is aware of how values are influenced by the different clinical scenarios presented in this article [5]. 
This study is a novel study that demonstrated predictive validity of PCT level as a single and instant approach to estimate in-hospital outcome of postsurgical trauma patients who likely to suffer sepsis and other bacterial infections. However, there is no single likewise study in literature to compare in the use of PCT level in PICU patients and best cutoff point to project in-hospital outcome. Our study also emerged an additional feature related to average survival time of patients as 25 days in ICU with a $90 \%$ probability of mortality within first 15 days, whereas average survival time was 17 days on mechanical ventilator and $95 \%$ probability of mortality in first 7-8 day. Sole-Rebalta et al described their limitation as these were only three deaths and could not be stratified before multiple organ failure to compare predictive validity related to outcome, however they reported an average stay in the PICU about 5 days and for the total length of hospitalization was 14.5 days [18].

Despite having various cutoff points of PCT level on various interval, there is no even a single study to compare and fix it as typical cutoff to be used as goldstandard biomarker for estimation of severity of in-hospital outcome. Another limitation of the study is retrospective design of study which does not confirm PCT level on a certain point was used to label severity of in-hospital outcome, rather a prospective cross-sectional study can confirm if based on elevated PCT level to some extent really indicated poor outcome and provided effect of an instant management plan based on single non-invasive serum biomarker.

\section{Conclusions}

Serum procalcitonin level as a single laboratory biomarker is remarkably useful in prediction of in-hospital outcome of pediatric patients after surgical infection for commencement of earliest treatment plan as lifesaving attempt. In a longer duration of hospital stay, a blended approach based on PCT level with other clinical biomarkers can be developed for monitoring of given treatment as well as switching to more effective remedies. The role of PCT level can be further explored in prospective cross sectional studies restricted to homogeneous post-surgical trauma category.

\section{List Of Abbreviations}

AUC - Area under the Curve

CRP - C - reactive protein

ICU - Intensive Care Unit

ISS - Injury Severity Score

NPV - Negative Predictive Values

PCT - Procalcitonin

PICU - Pediatric Intensive Care Unit

PLT - Pediatric Liver Transplant

PPV - Positive Predictive Values

PRISM - Pediatric Risk of Mortality Score

ROC - Receptive Operating Curve

SD - Standard Deviations

SIRS - Systematic Inflammatory Response Syndrome

\section{Declarations}

- Ethics approval and consent to participate: The author confirms this study was in accordance to institutional guidelines and regulation and confirms that informed consent was obtained from all participants and/or their legal guardians. The ethical approval for this study was obtained from the Institutional review board (IRB) of Imam Abdulrahman Bin Faisal University with an IRB number 2021-01-078

- Consent for publication: Not applicable

- Availability of data and materials' statement: The datasets used and/or analyzed during the current study are available from the corresponding author on reasonable request.

- Competing Interests: The author declares no competing interests.

- Funding: This research did not receive any specific grant from funding agencies in the public, commercial, or not-for-profit sectors.

- Author's contributions: The author solely made the study conception and design, data acquisition, analysis and data interpretation, drafting of the manuscript and critical revision.

- Acknowledgements: I would like to express my great thanks to Dr. Intisar Ahmad Siddiqui, Abdulrahman Bin Faisal University, College of Dentistry and Dr. Faisal Othman Alqurashi, College of Medicine for their willingness and support. Next, my great thanks also go to data collectors that support me throughout the work. Lastly, I would like to thank Editage (https://eur02.safelinks.protection.outlook.com/? 
url=http\%3A\%2F\%2Fwww.editage.com\%2F\&amp;data=04\%7C01\%7Cwbuali\%40iau.edu.sa\%7C92e52a111295478cdb0808d8f9b8caf9\%7C2c86bbfc8d04. for English language editing.

\section{References}

1. AlSomali A, Mobarki A, Almuhanna M, Alqahtani A, Alhawali Z, Almalki A, et al. Systemic inflammatory response syndrome after major abdominal surgery. IJMDC. 2019;3(1):113-116.

2. Al Ameer HH, AlKadhem SM, Busaleh F, AlKhwaitm S, Llaguno MB. Multisystem Inflammatory Syndrome in Children Temporally Related to COVID-19: A Case Report From Saudi Arabia. Cureus. 2020 Sep;12(9).

3. Matics TJ, Sanchez-Pinto LN. Adaptation and validation of a pediatric sequential organ failure assessment score and evaluation of the sepsis-3 definitions in critically ill children. JAMA pediatrics. 2017 Oct 1;171(10):e172352-

4. Cies JJ, Chopra A. Procalcitonin use in a pediatric intensive care unit. The Pediatric infectious disease journal. 2014 Sep 1;33(9):984-6.

5. Yunus I, Fasih A, Wang Y. The use of procalcitonin in the determination of severity of sepsis, patient outcomes and infection characteristics. PloS one. 2018 Nov 14;13(11):e0206527.

6. Aygun F. Procalcitonin value is an early prognostic factor related to mortality in admission to pediatric intensive care unit. Critical care research and practice. 2018 Dec 24;2018.

7. Deng S, Zhu H, Wang K, Cao T. Procalcitonin as a marker of sepsis and outcome in patients with neurotrauma: an observation study. BMC anesthesiology. 2013 Dec;13(1):1-6.

8. Gasim GI, Musa IR, Yassin T, Al Shobaili HA, Adam I. Sepsis in Buraidah Central Hospital, Qassim, Kingdom of Saudi Arabia. International journal of health sciences. 2016 Apr;10(2):175.

9. Albuali WH, Algamdi AA, Hasan EA, Al-Qahtani MH, Yousef AA, Al Ghamdi MA, Bubshait DK, Alshahrani MS, AlQurashi FO, Shahmah TA, Awary BH. Use of a Mortality Prediction Model in Children on Mechanical Ventilation: A 5-Year Experience in a Tertiary University Hospital. Journal of Multidisciplinary Healthcare. 2020;13:1507.

10. AlRawahi AN, AlHinai FA, Doig CJ, Ball CG, Dixon E, Xiao Z, Kirkpatrick AW. The prognostic value of serum procalcitonin measurements in critically injured patients: a systematic review. Critical Care. 2019 Dec;23(1):1-2.

11. Balci CA, Sivaci R, Akbulut G, Karabekir HS. Procalcitonin levels as an early marker in patients with multiple trauma under intensive care. Journal of International Medical Research. 2009 Dec;37(6):1709-17.

12. Masson et al. Presepsin (soluble CD14 subtype) and procalcitonin levels for mortality prediction in sepsis: data from the Albumin Italian Outcome Sepsis trial. Critical care. 2014 Feb;18(1):1-9.

13. Meisner M, Adina H, Schmidt J. Correlation of procalcitonin and C-reactive protein to inflammation, complications, and outcome during the intensive care unit course of multiple-trauma patients. Critical care. 2005 Feb;10(1):1-0.

14. Koutroulis I, Loscalzo SM, Kratimenos P, Singh S, Weiner E, Syriopoulou V, Theocharis S, Chrousos G. Clinical applications of procalcitonin in pediatrics: an advanced biomarker for inflammation and infection-can it also be used in Trauma?. International Scholarly Research Notices. $2014 ; 2014$.

15. Aydemir C, Aydemir H, Kokturk F, Kulah C, Mungan AG. The cut-off levels of procalcitonin and C-reactive protein and the kinetics of mean platelet volume in preterm neonates with sepsis. BMC pediatrics. 2018 Dec;18(1):1-2.

16. Sakran JV, Michetti CP, Sheridan MJ, Richmond R, Waked T, Aldaghlas T, Rizzo A, Griffen M, Fakhry SM. The utility of procalcitonin in critically ill trauma patients. Journal of Trauma and Acute Care Surgery. 2012 Aug 1;73(2):413-8.

17. Cousin VL, Lambert K, Trabelsi S, Galetto-Lacour A, Posfay-Barbe KM, Wildhaber BE, McLin VA. Procalcitonin for infections in the first week after pediatric liver transplantation. BMC infectious diseases. 2017 Dec;17(1):1-7.

18. Solé-Ribalta A, Bobillo-Pérez S, Valls A, Girona-Alarcón M, Launes C, Cambra FJ, Jordan I, Esteban E. Diagnostic and prognostic value of procalcitonin and mid-regional pro-adrenomedullin in septic paediatric patients. European journal of pediatrics. 2020 Jan 23:1-8.

\section{Figures}




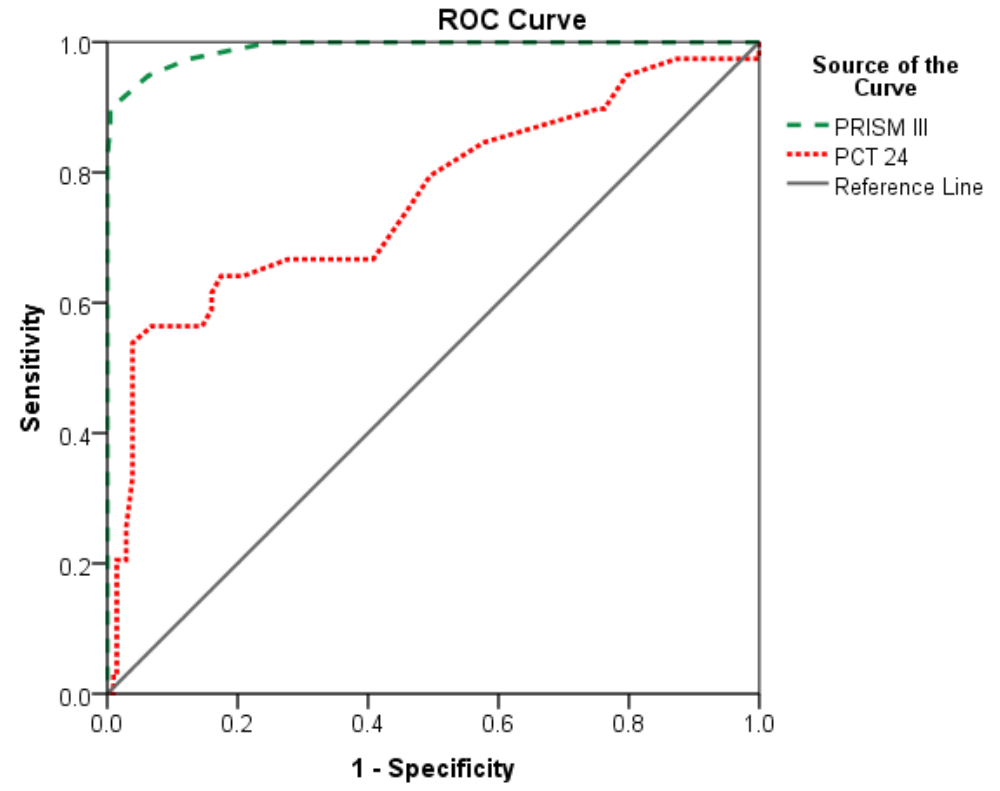

Diagonal segments are produced by ties.

\section{Figure 1}

PRISM III score AUC=0.99 (95\% Cl: 0.98-1.0), p<0.001 (High predictive validity) PCT 24 hours AUC=0.76 (95\% Cl: 0.66-0.85), p<0.001 (High predictive validity)

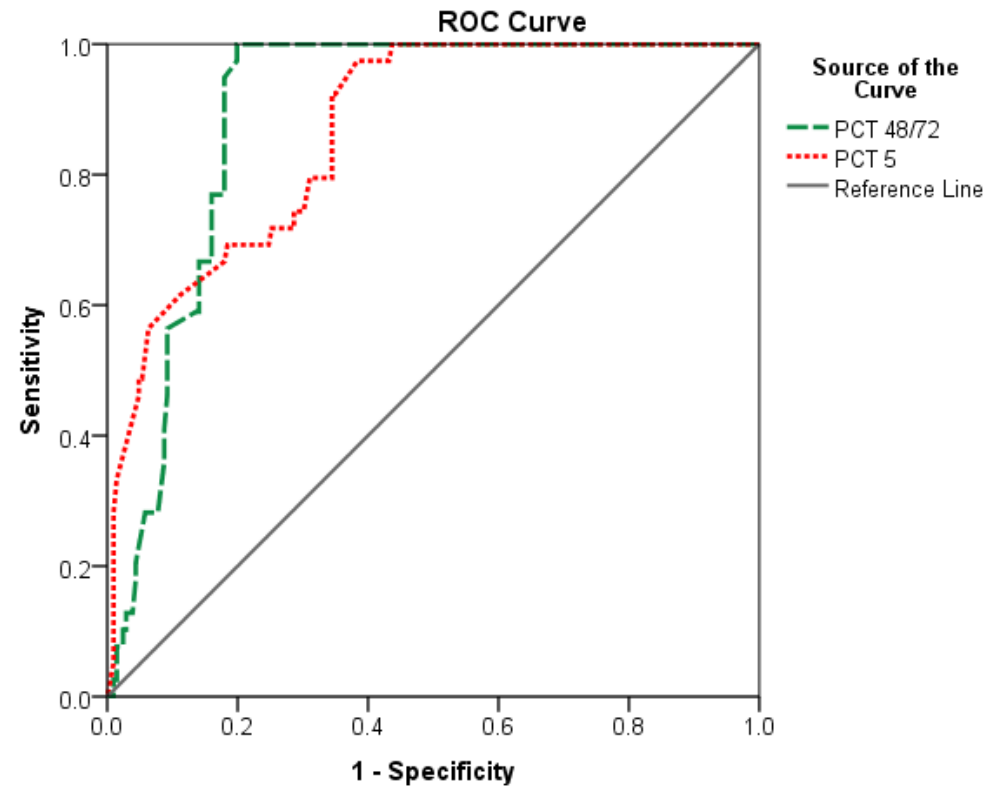

Diagonal segments are produced by ties.

Figure 2

PRISM III score AUC=0.99 (95\% Cl: 0.98-1.0), p<0.001 (High predictive validity) PCT 24 hours AUC=0.76 (95\% Cl: 0.66-0.85), p<0.001 (High predictive validity) 


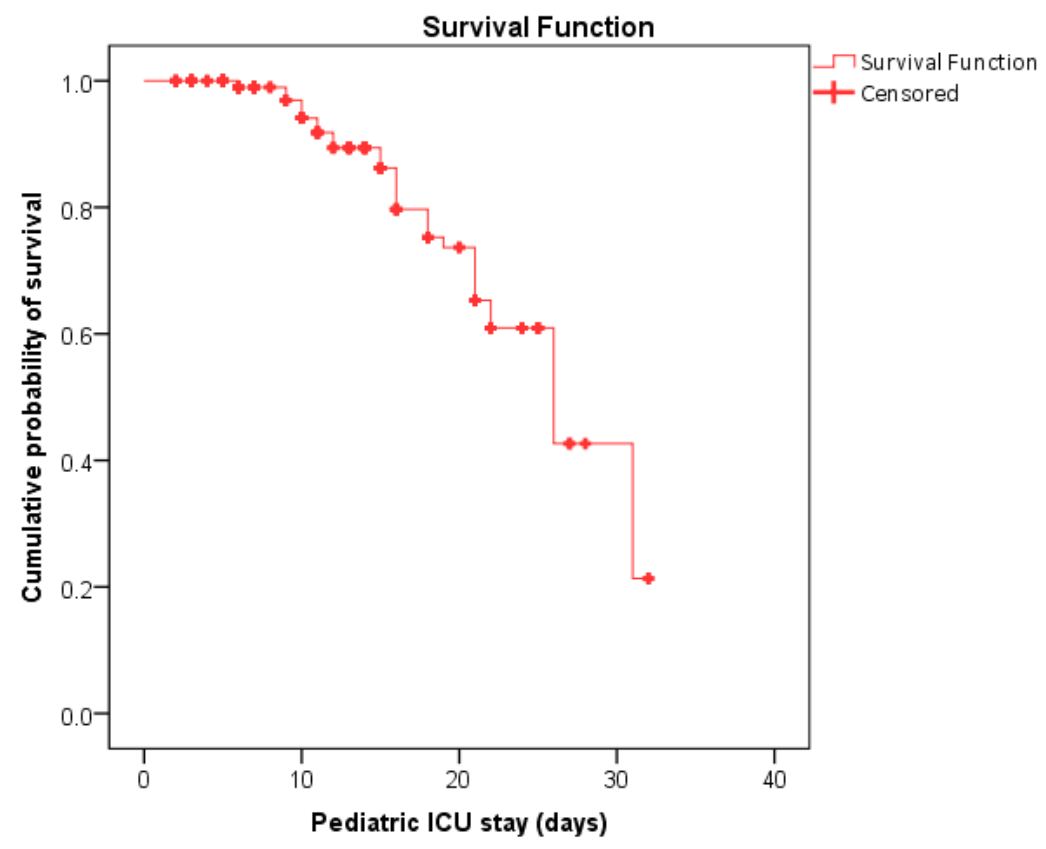

\section{Figure 3}

The mean survival time in PICU was 24.6 (95\% Cl: 22.8-26.3) days, highest probability of mortalities (90\% or above) within first 15 days

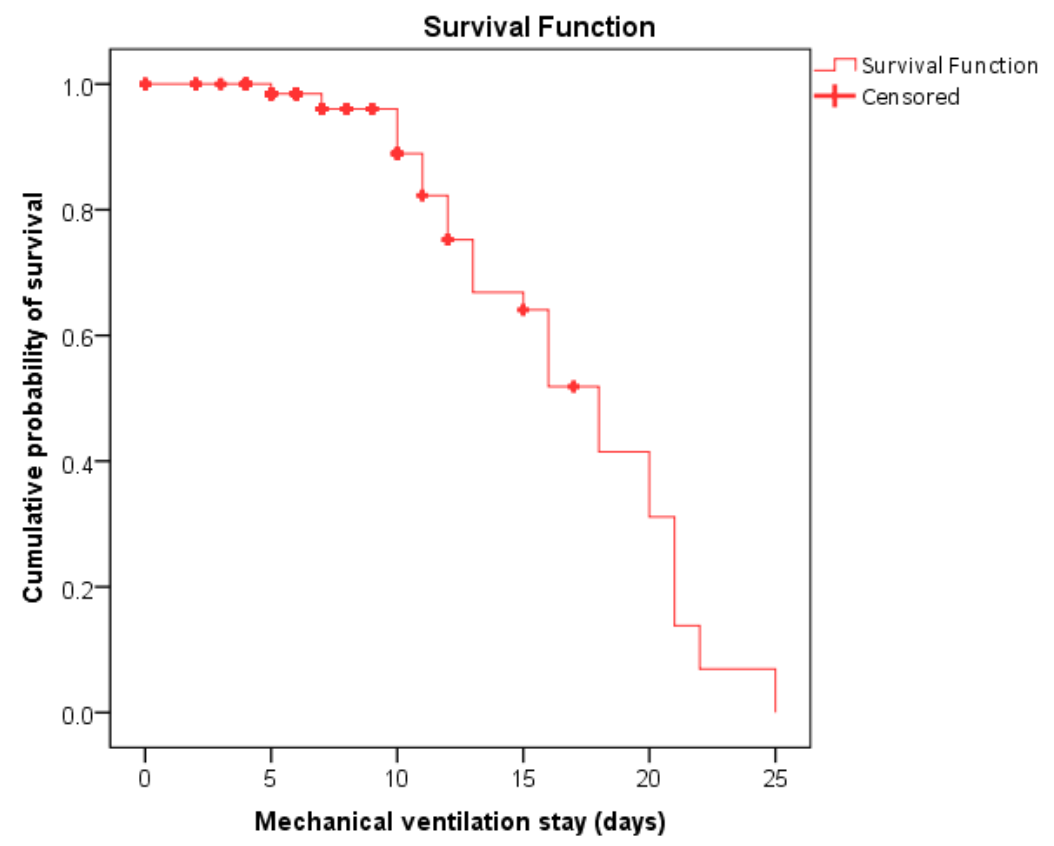

Figure 4

The mean survival time on mechanical ventilator was 16.8 (95\% Cl: 15.4-18.2) days, highest probability of mortalities (95\% or above) within first 7-8 days 